ISSN 1870-4069

\title{
Reconocimiento de rostros mediante estructuras faciales antropométricas
}

\author{
Yair Velasco-Ramírez, Edgardo M. Felipe-Riverón, Ricardo Barrón-Fernández \\ Instituto Politécnico Nacional, México \\ rbarron@cic.ipn.mx
}

\begin{abstract}
Resumen. Las estructuras faciales antropométricas son estructuras que se obtienen mediante puntos somatométricos (los cuales son empleados para la extracción de características craneofaciales). En este trabajo se propone una estructura facial antropométrica que contribuya al desarrollo de un método que realice reconocimiento de rostros de manera automática. Este método está basado principalmente en cinco etapas: extracción de coordenadas de los puntos somatométricos, creación de la estructura antropométrica, cálculo de distancias entre puntos, detección de ángulos de los puntos y cálculo de proporciones respecto a las distancias. Las distancias y los ángulos se obtienen con base en la relación de los puntos, relación que es establecida con la estructura antropométrica propuesta. A diferencia de otros trabajos relacionados con el reconocimiento de rostros, el método propuesto utiliza solamente nueve puntos, lo que es, hasta ahora, uno de los menores números de puntos utilizados.
\end{abstract}

Palabras clave: reconocimiento, rostros, puntos somatométricos, estructura antropométrica.

\section{Recognition of Faces using Anthropometric Facial Structures}

\begin{abstract}
Anthropometric facial structures are structures obtained through somatometric points (which are used for the extraction of craniofacial features). In this work we propose an anthropometric facial structure that contributes to the development of a method that performs face recognition automatically. This method is based mainly on five stages: extraction of coordinates of the somatometric points, creation of the anthropometric structure, calculation of distances between points, detection of angles of the points and calculation of proportions with respect to distances. Distances and angles are obtained based on the relationship of the points, a relationship that is established with the proposed anthropometric structure. Unlike other works related to the recognition of faces, the proposed method uses only nine points, which is, until now, one of the lowest number of points used.
\end{abstract}

Keywords: recognition, faces, somatometric points, anthropometric structure. 


\section{Introducción}

Todo sistema de reconocimiento de rostros requiere de una previa adquisición de características, mismas que sean capaces de brindar información sobre el rostro de una persona. A partir de dichas características es posible generar una alternativa para representar el rostro, la cual, para este trabajo, es una estructura antropométrica.

Esta estructura necesita cubrir al menos dos aspectos importantes: que sea capaz de representar un rostro y que dicha estructura sea única para cada persona.

Debido a que hay rasgos faciales que tienden a sufrir cambios con el paso del tiempo o pueden ser modificados por procedimientos menores (como maquillaje, barba, etc.), la estructura antropométrica que se propone está basada en nueve puntos somatométricos, mismos que fueron específicamente seleccionados debido a que son poco alterables inclusive bajo la influencia de algunas expresiones faciales.

Utilizando estos nueve puntos, se está realizando un trabajo de reconocimiento de rostros con un número pequeño de puntos, lo cual resulta una ventaja al momento de no contar con otros puntos visibles o, simplemente, que éstos no puedan ser obtenidos.

El propósito sustancial de utilizar este número de puntos es reducir redundancias a la hora de realizar la clasificación y, de igual manera, evitar procesar mucha información para el reconocimiento de un rostro.

El principal factor a considerar en el método propuesto para el reconocimiento de rostros es el conjunto de características o conjunto de patrones necesarios para realizar comparaciones entre rostros. Por esta razón, es necesario que para cada una de las imágenes la zona facial se muestre completa, es decir, que la persona esté de frente, esto es con la finalidad de evitar que alguno de los nueve puntos se pierda. De igual manera, las imágenes requieren tener un cierto nivel de iluminación, ya que si son demasiado obscuras podría no ubicarse un punto de manera correcta o, en el peor de los casos, omitirse por completo.

\section{Estado del arte}

Existen diferentes técnicas de reconocimiento facial; todas ellas extraen ciertas características del rostro para la etapa de clasificación. En el trabajo desarrollado por Dubey et al. [3] mencionan que el éxito de la metodología en el reconocimiento facial depende en gran medida de la selección de las características utilizadas por el sistema de reconocimiento. Proponen un método de selección de características a través de la Somatología (ciencia derivada de la Antropología) y sugieren tomar dos imágenes del sujeto para la extracción de 37 puntos: una frontal, de la que se obtienen 25 puntos, y otra de perfil, de la cual se extraen 12 puntos y con base en esos puntos, calculan 12 distancias euclidianas entre los puntos de la imagen frontal y 8 distancias más entre los puntos de la imagen de perfil. 
Gupta et al. [5] desarrollaron en el 2010 un algoritmo de reconocimiento facial en tres dimensiones que emplea distancias geodésicas y euclidianas en 3D para 10 puntos faciales somatométricos, llamado "Anthroface". Aislaron 70 proporciones antropométricas (propuestas por Farkas [4]) asociadas con la región facial, de las cuales identificaron que 23 tienen valores altos de desviación estándar para poblaciones de adultos. Se etiquetaron de manera manual los 25 puntos faciales asociados a las 23 proporciones identificadas. Con base en la experimentación, Gupta concluyó que algunos de los 25 puntos utilizados eran redundantes, por lo que se determinó un subconjunto de 10 puntos; al comparar los resultados del reconocimiento de los 25 puntos propuestos inicialmente y estos 10 puntos, los resultados fueron estadísticamente equivalentes, de modo que al final se utilizaron 10 de los 25 puntos propuestos.

La tabla 1 contiene trabajos sobre reconocimiento de rostros, mostrando los resultados obtenidos para cada uno, mismos que servirán como punto de comparación para el método propuesto en este trabajo.

Tabla 1. Resultados de trabajos afines.

\begin{tabular}{lc}
\hline Artículo & Resultados (accuracy) \\
\hline $\begin{array}{l}\text { Face detection and recognition with SURF for human-robot } \\
\text { interaction [2] }\end{array}$ & $96 \%$ \\
\hline Face detection using information fusion [1] & $93 \%$ \\
\hline Face Recognition Using Local Binary Decisions [6] & $91-96 \%$ \\
\hline Fast face recognition based on fractal theory [8] & $93.3 \%$ \\
\hline Applying artificial neural networks for face recognition [7] & $94.7-96.7 \%$ \\
\hline
\end{tabular}

\section{Desarrollo}

\subsection{Puntos somatométricos seleccionados}

El conjunto de puntos sobre el cual se basa la construcción de la estructura antropométrica (mediante observación y con base en el estudio realizado por Gupta) es considerado como el conjunto que aporta más información geométrica del rostro y que mejor lo caracteriza debido a la mínima variación que presentan los puntos ante expresiones faciales y ligeras rotaciones en los distintos planos: axial, coronal y sagital.

Los puntos somatométricos utilizados son: de la región de los ojos, los dos exocanthiones (ex, ex') y los dos endocanthiones (en, en'), así como el nasion (n); de la región de la nariz únicamente el subnasal (sn); y de la región de la boca el stomion (sto) y los dos goniones (go, go') (figura 1). 


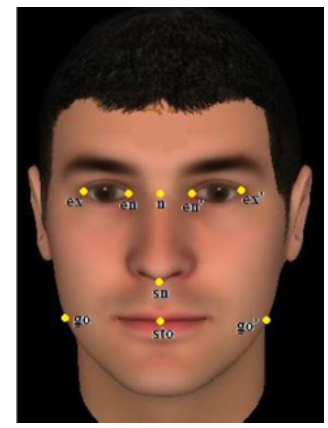

Fig. 1. Puntos somatométricos seleccionados.

\subsection{Obtención de las coordenadas de los puntos y estructura propuesta}

El trabajo realizado toma como principio que los puntos ya están ubicados en el rostro, por lo que para la obtención de las coordenadas, se procesa una imagen en escala de grises con los puntos somatométricos resaltados en rojo (figura 2a). Las coordenadas de los pixeles de los puntos se van almacenando en orden de aparición de derecha a izquierda (tomando como referencia la perspectiva del rostro en la imagen) de tal forma que faciliten la construcción de la estructura antropométrica (figura 2b).

El propósito principal es, entonces, establecer patrones a partir de la estructura que permitan establecer un margen de similitud o diferencia bajo comparaciones entre dichos patrones para poder realizar el reconocimiento de un rostro mediante un algoritmo de clasificación.

La estructura antropométrica está conformada por los puntos (nodos) y las distancias entre estos (aristas). Tomando el supuesto que los rostros que se utilizarán para los experimentos son simétricos, se plantea una relación entre nodos donde, si se divide la estructura exactamente por la mitad de tal manera que solo tendrán relación aquellos puntos que se encuentren del lado derecho con los puntos del lado derecho y los que se encuentren del lado izquierdo tendrán relación solamente con los del lado izquierdo.

Corrección en el plano sagital Las invariantes de rotación en los planos representan un problema a la hora de extraer las mediciones de la estructura, ya que para poder obtener resultados satisfactorios es necesario que el rostro esté de frente, por lo que es preciso corregirlas antes de su cálculo.

Para la corrección de rotación del plano sagital, se toman en cuenta las coordenadas de los dos endocanthiones. Se obtiene la pendiente de la línea generada entre el endocanthion derecho y el endocanthion izquierdo. El ángulo de rotación del rostro es determinado por el arco tangente de la pendiente $(\mathrm{a}, \mathrm{b})$ 

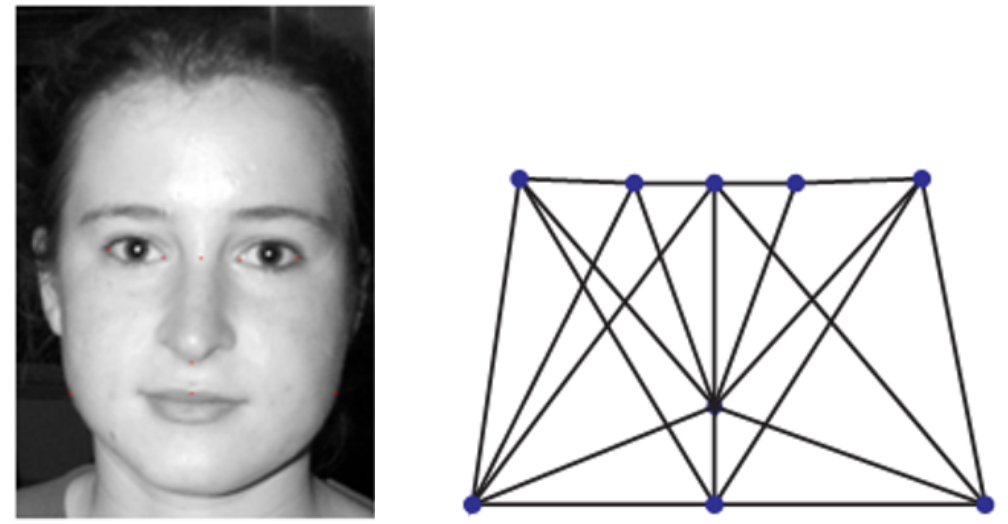

Fig. 2. a) Puntos ubicados en el rostro, b) Estructura antropométrica utilizada.

(ecuación 1):

$$
\theta=\tan ^{-1}\left(\frac{b_{2}-b_{1}}{a_{2}-a_{1}}\right)
$$

En un plano de coordenadas $\mathrm{x}$, y la rotación de una imagen se obtiene mediante el producto de una matriz de rotación por el vector correspondiente a las coordenadas $[\mathrm{x}, \mathrm{y}]$ de cada uno de los pixeles de la imagen. Se debe tener en cuenta que para una imagen el eje $\mathrm{x}$ corresponde a las filas $\mathrm{y}$ el eje y a las columnas, por tanto, las coordenadas de los pixeles de la imagen rotada responden a la ecuación 2 :

$$
\begin{aligned}
& x^{\prime}=y \cdot \sin \theta+x \cdot \cos \theta, \\
& y^{\prime}=y \cdot \cos \theta-x \cdot \sin \theta .
\end{aligned}
$$

Corrección en el plano coronal La rotación en el plano coronal implica un problema debido a que produce una asimetría en la estructura antropométrica. Esta asimetría tiene dos implicantes; la primera, que la coordenada y del nasion es diferente a la coordenada y del subnasal y el stomion. Puesto que, al tener el rostro de frente, la distancia entre el gonion derecho y el stomion debe ser semejante a la distancia entre el gonion izquierdo y el stomion. La segunda implicante que presenta la asimetría de la estructura antropométrica es la diferencia de distancia entre estos puntos en el eje x. De modo que, si el rostro está rotado hacia la derecha, la distancia entre el gonion izquierdo y el stomion es mayor que la distancia entre el gonion derecho y el stomion (figura 3).

La diferencia entre las distancias del gonion izquierdo-stomion y gonion derecho-stomion se establece con una diferencia máxima de pixeles. Si dicha tolerancia es superada existe una rotación del rostro en el plano coronal.

A diferencia de la corrección del ángulo de rotación sobre el plano sagital, la corrección sobre el plano coronal no se realiza sobre la imagen, esta corrección 

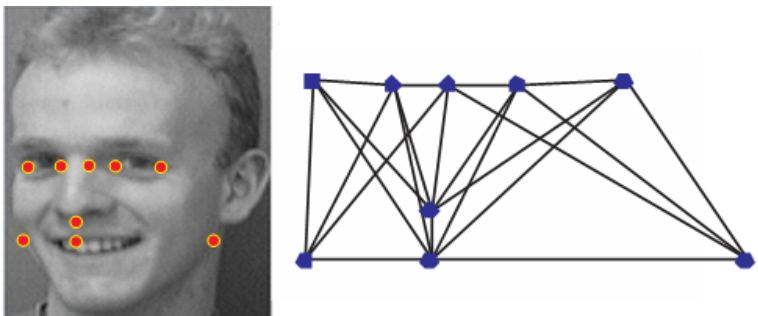

Fig. 3. El plano coronal.

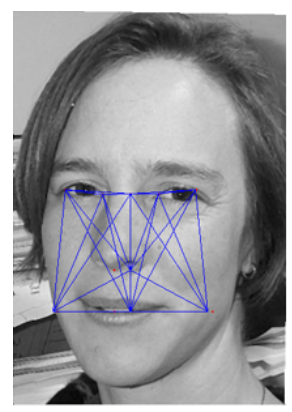

Fig. 4. Corrección de la rotación en el plano coronal.

se realiza únicamente modificando las coordenadas $\mathrm{x}$ de todos los puntos de la estructura antropométrica, con excepción del nasion, ya que éste es tomado como referencia. Para poder establecer las nuevas coordenadas de los puntos se calcula la distancia promedio entre puntos pares (gonion izquierdo - gonion derecho, etc.)

Posteriormente, tomando como referencia la coordenada $\mathrm{x}$ del nasion, se reubican los puntos a la distancia calculada y se actualizan las coordenadas. En el caso del stomion y subnasal, quienes no son considerados como puntos pares, sus nuevas coordenadas son establecidas por la coordenada $\mathrm{x}$ del nasion. En la figura 4 se puede ver la reubicación de los puntos sobre la imagen, así como los puntos originales.

Obtención de los patrones La estructura antropométrica propuesta permite obtener diversas características que pueden ser patrones para el algoritmo de clasificación, sin embargo, no todas son útiles ya que algunas poseen menos información que caracterizan al rostro inconsistentemente, es decir, puede haber ciertas características que no se presten para realizar comparaciones entre las mismas ya que no se pueden normalizar o sus valores varían demasiado entre diversas imágenes del rostro de una misma persona. Por este motivo se seleccionó un conjunto de tres características que, con base en los experimentos realizados, 
permiten establecer un rango de error entre mediciones y, al mismo tiempo, establecer un margen de diferencia entre rostros de personas distintas. Las características seleccionadas son:

- Distancias entre puntos. Con base en la relación de puntos en la estructura antropométrica propuesta, se obtiene la distancia euclidiana de cada arista una de las aristas.

- Ángulo de la pendiente. El ángulo de la pendiente de cada una de las aristas se obtiene con un criterio distinto a las distancias. Se toma como origen al subnasal y se calculan las pendientes de aquellas aristas que sean adyacentes a este punto. Son un total de 9 mediciones más dos que corresponden a la inclinación de los ojos.

- Proporciones de distancias.Para resolver la invariante del tamaño del rostro se calculan proporciones de las distancias obtenidas. Una proporción equivale a dividir la distancia de cada arista entre el alto del rostro, de esta manera se asegura que todas las distancias de un mismo rostro varían en una mínima cantidad y así poder establecer una métrica más precisa de comparación entre personas.

Es importante resaltar que, a pesar de que las proporciones se calculan a partir de las distancias, las dos características de la estructura aportan información importante y se valoran de manera distinta en el clasificador.

Clasificación La base de datos cuenta con 470 imágenes de rostros que corresponden a 27 personas diferentes. A fin de comprobar que los patrones formados representan de manera precisa a un rostro, se utilizaron tres clasificadores:

- KNN,

- Clasificador bayesiano,

- MLP.

A fin de comprobar que los patrones formados representan de manera precisa a un rostro, se utilizaron tres clasificadores, dos de ellos probabilísticos ya que, al tener diversas clases con diferente número de elementos entre ellas, es posible establecer una función de densidad o directamente la probabilidad de que un elemento $x$ pertenezca a la clase $C_{j}$.

\section{Resultados}

\subsection{Corrección de invariantes y cálculo de mediciones}

La rotación de los rostros se soluciona de manera satisfactoria. En la figura 5 se muestra la corrección de la rotación del plano sagital para 4 rostros diferentes.

De igual manera, la corrección del plano coronal es satisfactoria. En la figura 6 se muestra la reubicación de los puntos para dos personas distintas y la estructura antropométrica sobre el rostro, esto con fin de apreciar la reestructuración de la misma. 


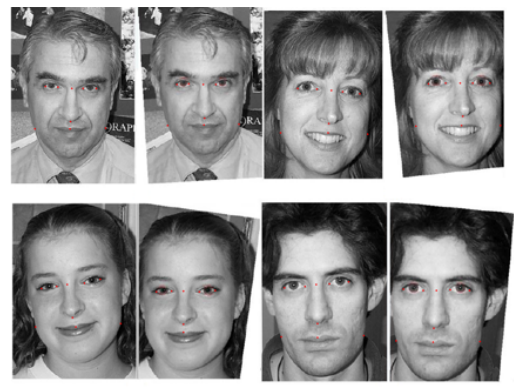

Fig. 5. Corrección de la rotación en el plano sagital.

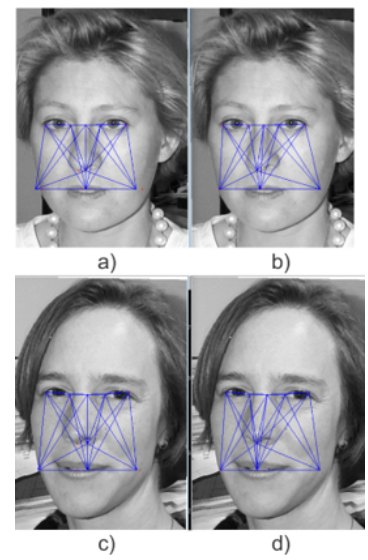

Fig. 6. Corrección del plano coronal, a) y c) corresponden a las imágenes originales, b) y d) corresponden a los puntos y la estructura corregida.

La corrección de la escala se probó en dos escenarios. El primero comparando las proporciones de la imagen de un rostro contra la misma imagen, solo que a esta última se le aplicó una reducción de tamaño del $30 \%$.

El segundo escenario fue comparar la imagen del rostro con otra imagen de la misma persona. Al final se obtienen mediciones bastante cercanas entre ellas (figura 7).

Los ángulos de las pendientes de los puntos se calculan planteando un sistema de coordenadas donde el subnasal es el origen (figura 8). Posteriormente, todas aquellas aristas que sean adyacentes a ese punto son procesadas. Aunados a estos puntos, están dos ángulos más, los ángulos que corresponden a las pendientes de los ojos. Estos últimos ángulos son procesados de manera independiente ya que no son adyacentes al subnasal, sin embargo, proporcionan información relevante del rostro. 


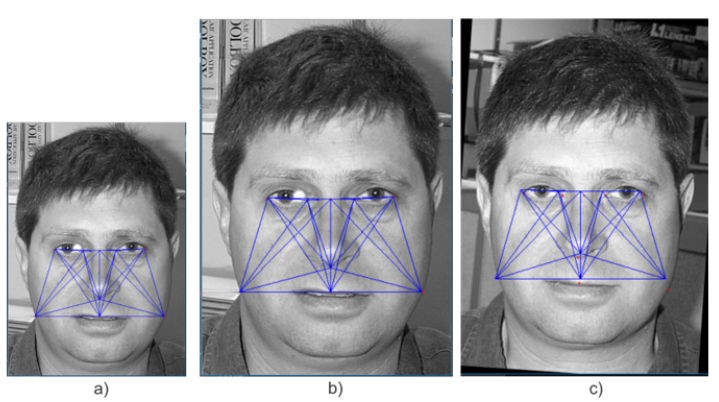

Fig. 7. Imágenes del rostro a comparar, a) reducción de la imagen, b) al $30 \%$, c) diferente imagen del mismo rostro.

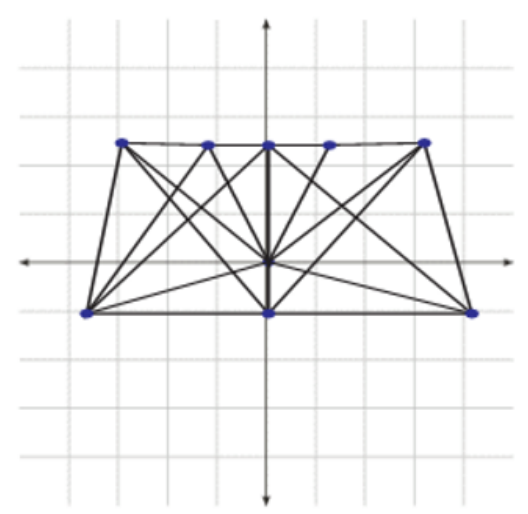

Fig. 8. Sistema de coordenadas.

\subsection{Clasificación}

Para las pruebas en la etapa de clasificación se utilizaron un total de 22 personas con una promedio de 20 imágenes para cada una, esto es debido a que algunas personas poseen un número bajo de imágenes para poder clasificarlas o, la iluminación de las mismas no permiten una adecuada localización de los puntos. Por tanto, se utilizó un total de 446 imágenes, donde se aplicó el $70 \%$ para entrenamiento y el $30 \%$ para prueba. Respecto al clasificador del vecino más cercano, se utilizó una configuración donde se establece un $\mathrm{k}=5$. Las mediciones de recall, presicion y accuracy se muestran en la tabla 2 .

\section{Conclusiones}

Se observan resultados por debajo del promedio de los trabajos afines, por lo que es hay dos posibles alternativas. Replantear las características propuestas 
Tabla 2. Resultados de la clasificación de los rostros con los dos clasificadores.

\begin{tabular}{cccc}
\hline \hline \multicolumn{4}{c}{ KNN Bayesiano MLP } \\
\hline Presicion & 64.5 & 68.88 & 68.33 \\
\hline Recall & 61.32 & 70 & 73.33 \\
\hline Accuracy & 62 & 71.66 & 75.5 \\
\hline
\end{tabular}

o, en su defecto, incrementarlas añadiendo áreas significativas o alguna otra característica que pueda brindar información del rostro. La segunda, probar con otro tipo de clasificador en donde se puedan ponderar los pesos o la importancia que se le da a cada característica.

A pesar de que los resultados son bajos, los resultados que arroja el clasificador bayesiano y la MLP son más altos que los del clasificador KNN. Es probable que, con base en estas observaciones, la solución más factible pudiera ser el proponer un clasificador más robusto.

Por otro lado, los resultados que se obtienen con el clasificador KNN indican que las mediciones entre rostros de personas diferentes sean muy similares. Hay que establecer un margen de diferencia más preciso para poder tener mayor distinción entre rostros.

Agradecimientos. Proyectos SIP 20181698, 20181895.

\section{Referencias}

1. Aarabi, P., Lam, J.C.L., Keshavarz, A.: Face detection using information fusion. In: Information Fusion, 10th International Conference on. pp. 1-8 (2007)

2. An, S., Ma, X., Song, R., Li, Y.: Face detection and recognition with surf for human-robot interaction. In: Automation and Logistics. ICAL'09. IEEE International Conference on. pp. 1946-1951 (2009)

3. Dubey, S., Sharma, T.: A face recognition system through somatology. International Journal on Computer Science and Engineering 3(1), 155-160 (2011)

4. Farkas, L., Munro, I.: Anthropometric facial proportions in medicine. Thomas Books, first edn. (1987)

5. Gupta, S., Markey, M., Bovik, A.: Anthropometric 3d face recognition. International Journal of Computer Vision 90(3), 331-349 (2010)

6. James, A.P., Dimitrijev, S.: Face recognition using local binary decisions. IEEE Signal Processing Letters 15, 821-824 (2008)

7. Le, T.H.: Applying artificial neural networks for face recognition. Advances in Artificial Neural Systems 15 (2011)

8. Tang, Z., Wu, X., Fu, B., Chen, W., Feng, H.: Fast face recognition based on fractal theory. Applied Mathematics and Computation 321, 721-730 (2018) 